\title{
Aktuelle Rechtsfragen der Genehmigungspraxis in der Teleradiologie
}

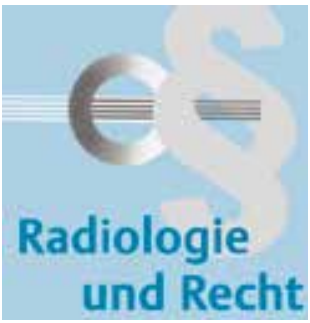

\section{Einführung}

$\S 3$ Absatz 4 der Röntgenverordnung (RöV) eröffnet unter bestimmten Voraussetzungen die Möglichkeit zum Einsatz teleradiologischer Systeme für die Anwendung von Röntgenstrahlung zur Untersuchung von Menschen. Wie auch in allen anderen Anwendungsfällen von Röntgenstrahlung am Menschen bedarf die Teleradiologie einer rechtfertigenden Indikation nach $\S 23$ Abs. 1 RöV. Jedoch muss sich der für das Stellen der rechtfertigenden Indikation befugte Arzt nicht - wie in sonstigen Anwendungsfällen - am Ort der Untersuchung oder in dessen unmittelbarer Nähe aufhalten (s.a. §2 Nr.25, §23 Abs. 1 Satz 5 RöV).

In vielen Anwendungsfällen ergeben sich hieraus Vorteile für den Patienten. Zeitliche Verzögerungen bei Untersuchungen und insbesondere Transportrisiken können durch die Zulassung der Teleradiologie reduziert werden. Andererseits kann die größere räumliche Entfernung des die rechtfertigende Indikation stellenden Arztes vom Ort der Untersuchung zu Nachteilen für den Patienten, insbesondere zu im Einzelfall ungerechtfertigten Strahlenexpositionen führen.

Der Einsatz teleradiologischer Systeme ist nach § 4 Abs. 4 Nr. 3 RöV immer genehmigungspflichtig, also auch bei Vorliegen der Voraussetzungen nach $\S 4$ Abs. 1 RöV. Um zu verhindern, dass die Anwendung der Teleradiologie dazu führt, dass an einem Krankenhaus generell keine Person nach $\S 24$ Abs. 1 Nr. 1 oder 2 RöV mehr vorhanden ist, soll die Teleradiologie grundsätzlich nur für den Fall des Nacht- oder Wochenenddiensts zulässig sein, da eine durchgehende Versorgung mit entsprechend fachkundigem Personal in der Regel nicht gewährleistet werden kann. Ausnahmsweise kann die zuständige Behörde nach Satz 2 den Einsatz der Teleradiologie auch für den Tagesbetrieb zulassen.
Zur Erteilung der Genehmigung sind in jedem Fall die in §3 Absatz 4 Satz 1 RöV aufgeführten Voraussetzungen zusätzlich zu denen der sonstigen Anwendungsfälle von Röntgenstrahlung am Menschen zu erfüllen. Hiernach darf die rechtfertigende Indikation ausschließlich von einem Arzt mit umfassender Fachkunde im Strahlenschutz nach $§ 23$ Abs. 1 Nr. 1 RöV gestellt werden, der auch die Befundung vorzunehmen hat und damit die Gesamtverantwortung für die Anwendung der Röntgenstrahlung am Menschen übernimmt. Dieser ist im Falle der Teleradiologie der „anwendende Arzt“ im Sinne der $\S \S 23$ ff. RöV. Für die technische Seite der Anwendung muss zumindest eine Person nach § 24 Abs. 2 Nr. 1 oder 2 RöV zur Verfügung stehen. Mit dem Begriff „zumindest“ soll zum Ausdruck gebracht werden, dass selbstverständlich auch eine höher qualifizierte Person die Röntgenuntersuchung vor Ort durchführen darf, wenn sie über die erforderliche Fachkunde im Strahlenschutz verfügt; nicht zugelassen werden soll hier eine Person nach §24 Abs. 2 Nr. 3 RöV.

Erforderlich ist darüber hinaus in jedem Fall die Anwesenheit eines Arztes mit Kenntnissen im Strahlenschutz. Falls also am Untersuchungsort eine MedizinischTechnische Röntgenassistentin oder ein Medizinisch-Technischer Röntgenassistent die Röntgenuntersuchung technisch durchführt und kein Arzt mit Fachkunde im Strahlenschutz vor Ort ist, muss dort sichergestellt sein, dass ein anderer Arzt für medizinisch notwendiges Handeln unmittelbar zur Verfügung steht. Dieser muss zumindest über die Kenntnisse im Strahlenschutz verfügen, die es ihm ermöglichen, die Vorgaben des verantwortlichen Arztes nachzuvollziehen und gemeinsam mit der oder dem MTRA die Untersuchung durchzuführen.

Eine unmittelbare Zusammenarbeit zwischen dem Arzt nach §3 Absatz 4 Satz 1 Nr.1 RöV einerseits und der oder dem MTRA sowie dem Arzt am Untersu- 
chungsort des Patienten andererseits muss organisatorisch und technisch sichergestellt sein. Der fachkundige Arzt muss somit unter Mitwirkung der Personen am Untersuchungsort alle für die rechtfertigende Indikation erforderlichen Informationen erlangen und darüber hinaus auch während der Untersuchung des Patienten erforderlichenfalls Einfluss nehmen können. Dies setzt die Ausnutzung aller technischen Möglichkeiten der Telekommunikation voraus, also auch beispielsweise den Aufbau einer Videokonferenzschaltung. Weiterhin wird die Qualitätssicherung der technischen Komponenten des teleradiologischen Systems einschließlich der Übertragungswege vorausgesetzt, die durch Abnahmeprüfungen von einem Sachverständigen nach $\S 4$ a RöV und durch Nachweis der Funktionsfähigkeit des Datenübertragungssystems zu erbringen ist. Die Regelung des Absatz 4 Satz 1 Nr. 5 soll vor allem das Risiko des Patienten in besonders komplizierten Untersuchungsfällen reduzieren. Sie wirkt aber auch einer überregionalen Ausweitung teleradiologischer Kommunikationssysteme entgegen, die persönliche Kontakte zwischen dem Arzt mit der erforderlichen Fachkunde im Strahlenschutz und dem Arzt am Ort der Untersuchung zum Nachteil des Patienten ausschließen könnte (vgl. Entwurf einer Verordnung zur Änderung der Röntgenverordnung, Stand: Juli 2001).

Die Teleradiologie ist eine Ausnahme von dem Grundsatz des $§ 23$ Abs. 1 Satz 5 RöV, der für den Normalfall der Röntgenuntersuchung festlegt, dass der verantwortliche Radiologe anwesend ist und nicht nur die für die Anwendung der Röntgenstrahlung rechtfertigende Indikation stellt und die Untersuchungsergebnisse befundet, sondern auch die Verantwortung für die technische Durchführung übernehmen kann (VG Köln, Urt. v. 28.01.2010, Az.: 13 K 1158/06).

In Genehmigungsverfahren ergeben sich dabei häufig Streitpunkte bei den Voraussetzungen des teleradiologischen Betriebs einer Röntgeneinrichtung, insbesondere beim Einsatz im Tagesdienst sowie bei der Anzahl und Qualifikation der notwendigen Ärzte. Dabei ergibt sich insbesondere die Situation, dass bei den Genehmigungsbehörden unterschiedliche Rechtsansichten über die Auslegung der Anforderungen zur Genehmigung des teleradiologischen Betriebes nach der RöV bestehen. Darüber hinaus sind aktuell Frage- stellungen infolge der Einführung des Patientenrechtegesetzes vom 20.03.2013 (BGBl. I., S.277 ff.) entstanden.

\section{Begriff und Voraussetzungen der Teleradiologie $\checkmark$}

Die Teleradiologie wird in $\$ 2 \mathrm{Nr}$.24 RöV legaldefiniert. Danach stellt die Teleradiologie die Untersuchung eines Menschen mit Röntgenstrahlung unter der Verantwortung eines Arztes nach $\S 24$ Abs. 1 Nr. 1 RöV dar, der sich nicht am Ort der technischen Durchführung befindet, und der mithilfe elektronischer Datenübertragung und Telekommunikation insbesondere zur rechtfertigenden Indikation und Befundung unmittelbar mit den Personen am Ort der technischen Durchführung in Verbindung steht. Der teleradiologie Betrieb bedarf dabei immer einer Genehmigung. §3 Abs. 4 RöV stellt insoweit zusätzliche Genehmigungsvoraussetzungen auf, die über die normalen Genehmigungsvoraussetzungen für Röntgeneinrichtungen nach §3 Abs. 2 RöV hinausgehen. Diese Genehmigungspflicht wird von der Rechtsprechung auch im Hinblick darauf, dass diese für die ausführenden Radiologen und Krankenhäuser einen Eingriff in die Berufsfreiheit nach Art. 12 Abs. 1 GG darstellt, aufgrund der möglichen Schäden für die Gesundheit der Patienten durch eine zu hohe Strahlenbelastung für verfassungsgemäß erachtet (VG Köln, Urt. v. 28.01.2010, Az.: 13 K 1158/06).

\section{Teleradiologie im Tagesdienst}

Die Teleradiologie ist gemäß §3 Abs. 4 S. 2 RöV grundsätzlich auf den Betrieb in der Nacht sowie an Feiertagen und Wochenenden zu beschränken. Darüber hinaus kann gemäß §3 Abs. 4 S. 3 RöV auch der teleradiologische Betrieb im Tagesdienst angeordnet werden, wenn ein Bedürfnis im Hinblick auf die Patientenversorgung besteht. Dadurch ist die Teleradiologie im Tagesdienst in zweierlei Weise durch diesen Mischtatbestand beschränkt. Zum einen muss der unbestimmte Rechtsbegriff des Bedürfnisses bejaht werden, zum anderen hat die Behörde selbst bei Vorliegen eines Bedürfnisses ein Ermessen, ob sie die Genehmigung erteilt (VG Aachen, Urt. v. 08.02.2007; Az.: 6 K 276/06).

\section{Bedürfnisprüfung}

Nach Ansicht des Verwaltungsgerichts Aachen ist vor allem das Bedürfnis im Hin- blick auf die Patientenversorgung in der Praxis streitig, da meist eine Genehmigung für den Nacht-, Wochenend- und Feiertagsdienst bereits erteilt worden sei. Um dieses zusätzliche Bedürfnis festzustellen, greift das Gericht auf den Arbeitsentwurf zur - bisher nicht umgesetzten - Richtlinie zur Teleradiologie nach $\S 3$ Abs. 4 RöV zurück.

Entscheidend sind danach für das Verwaltungsgericht Aachen Kriterien wie besonders abgelegene Untersuchungsorte (z. B. Inseln), organisatorisch bedingte Ausnahmesituationen bei grundsätzlicher Versorgung durch einen Arzt mit der erforderlichen Fachkunde im Strahlenschutz am Untersuchungsort oder keine ortsnahe, den Patienten zumutbare fachkundige radiologische Versorgung oder Notfallversorgung am Krankenhaus unter Berücksichtigung der regionalen stationären und ambulanten Einrichtungen.

Weiterhin könnten folgende Kriterien herangezogen werden:

- „Ausmaß des Mangels an Ärzten mit der erforderlichen Fachkunde im Strahlenschutz zur radiologischen $\mathrm{Pa}-$ tientenversorgung am Untersuchungsort und in der Region,

- bisherige Bemühungen zur Lösung des Mangels an Ärzten mit der erforderlichen Fachkunde im Strahlenschutz ohne Einsatz der Teleradiologie,

- Entfernung zwischen Untersuchungsort und Aufenthaltsort des Teleradiologen (z. B. innerhalb kurzer Zeit, bis 30 Minuten, durch Teleradiologen erreichbar),

- für Teleradiologie vorgesehene Untersuchungsfrequenz (z. B. bei CT-Untersuchungen nicht mehr als 5 teleradiologische Untersuchungen pro Tag),

- für Teleradiologie vorgesehene Untersuchungsarten (z.B. Begrenzung auf weitgehend standardisierte Diagnostik mit geringer Strahlenexposition oder auf bestimmte Fragestellungen und Untersuchungsarten mit klarere medizinischer und rechtfertigender Indikation),

- Prozentsatz der teleradiologisch erbrachten Röntgenanwendungen an einer Röntgeneinrichtung (z. B. kleiner als $30 \%$ der insgesamt durchgeführten Röntgenanwendungen),

- Erfahrung des Teleradiologen hinsichtlich des für die Teleradiologie vorgesehenen Untersuchungsspektrums (z. B. durch Vorlage der vom Teleradiologen durchgeführten, für die Teleradiologie- 
anwendung relevanten Untersuchungszahlen der letzten 2 Jahre),

- über den Stand der Technik hinausgehende Funktionen und Qualitätssicherungsmaßnahmen am Teleradiologiesystem (z.B. Videokonferenzsystem, schnelle Datenleitung, Qualitätsmanagementsystem),

- Anbindung relevanter therapeutischer Einrichtungen, um die Bereitstellung der Bilder für die Weiterbehandlung und die schnelle Nutzung der durch die Röntgenanwendung erworbenen Informationen zu verbessern (z. B. Neurochirurgie)." (vgl. VG Aachen, Urt. v. 08.02.2007; Az.: 6 K 276/06)

\section{Restriktive Auslegung}

Das Gericht betont jedoch, dass der Bedürfnisbegriff restriktiv auszulegen sei, da erkennbar eine Stufenfolge der Genehmigung (teleradiologischer) Röntgeneinrichtungen in der RöV zu erkennen sei. Die Teleradiologie bleibe eine „Ersatzlösung“, da bei einer vollständigen Öffnung die Gefahr bestünde, dass künftig gar keine Ärzte mit der Fachkunde im Strahlenschutz in den Kliniken tätig seien. Dies bedeutet, dass es auch keine grundsätzliche Genehmigung der Teleradiologie in der Anwendung bei Notfällen, außerhalb der vom Verordnungsgeber definierten Fälle des Nacht-, Wochenend- und Feiertagsdienstes geben könne (vgl. auch OVG Nordrhein-Westfalen, Beschl. v. 18.12.2007; Az.: 20 A 943/07).

\section{Verantwortliche Ärzte im Rahmen der Teleradiologie}

Im Rahmen der Teleradiologie nach der RöV sind mindestens zwei Ärzte verantwortlich zu beteiligen. Dies ist zum einen der Teleradiologe nach $\S 3$ Abs. 4 S. 1 Nr. 1 RöV und zum anderen ein weiterer Arzt am Ort der technischen Durchführung nach §3 Abs. 4 S. 1 Nr. 3 RöV.

\section{Der Begriff des Teleradiologen}

Der Teleradiologe ist nach $\S 3$ Abs. 4 S. 1 Nr. 1 RöV eine Person nach $\S 24$ Abs. 1 Nr. 1 RöV, die sich nicht am Ort der technischen Durchführung der Untersuchung befindet und die rechtfertigende Indikation nach §23 Abs. 1 RöV für die Anwendung von Röntgenstrahlung am Menschen stellt, die Untersuchungsergebnisse befundet und die ärztliche Verantwortung für die Anwendung der Röntgenstrahlung trägt. §24 Abs. 1 Nr. 1 RöV meint dabei diejenigen
Personen, die als Ärzte approbiert sind oder denen die Ausübung des ärztlichen Berufs erlaubt ist und die für das Gesamtgebiet der Röntgenuntersuchung oder Röntgenbehandlung die erforderliche Fachkunde im Strahlenschutz besitzen. Der Teleradiologe muss somit die volle Fachkunde im Strahlenschutz innehaben.

\section{Der Arzt am Ort der technischen Durchführung}

Der 2. notwendige Arzt muss sich gemäß §3 Abs. 4 S. 1 Nr.3 RöV am Ort der technischen Durchführung der teleradiologischen Untersuchung befinden. Dieser ist nicht dafür zuständig, die rechtfertigende Indikation nach §23 RöV zu erstellen. Daher muss diese Person nicht die Fachkunde im Strahlenschutz innehaben. Ihre Tätigkeit beschränkt sich darauf, die zur Feststellung der rechtfertigenden Indikation erforderlichen Angaben zu ermitteln. Daher benötigt sie nur die erforderlichen Kenntnisse im Strahlenschutz, um dieser Aufgabe nachkommen zu können. Es handelt sich bei dieser Person nicht um einen Radiologen, sondern um einen Facharzt eines anderen Fachgebiets in der Einrichtung, die die teleradiologische Leistung einfordert.

Die Erlangung der erforderlichen Kenntnisse im Strahlenschutz kann durch einen Kurs bei der Ärztekammer erfolgen. Genauere gesetzliche Anforderungen zum Inhalt dieser Kenntnisse existieren jedoch nicht. Dabei ist zu beachten, dass der Arzt nach §3 Abs. 4 S. 1 Nr. 3 RöV keine Fachgebietsidentität mit der die Röntgenuntersuchung anfordernden Fachabteilung bzw. dem anfordernden Facharzt haben muss:

„Erfüllt ist wohl auch die Voraussetzung des $\S 3$ Abs. 4 S. 1 Nr.3 RöV, weil nach den Angaben der Kläger die am Ort der technischen Durchführung der CT-Untersuchungen anwesenden Krankenhausärzte Dr. ..., ... und ... in ihrem jeweiligen Teilgebiet Fachkunde im Strahlenschutz besitzen, also erst recht das in $\S 3$ Abs. 4 S. 1 Nr.3 RöV vorausgesetzte Tatbestandsmerkmal der „erforderlichen Kenntnisse im Strahlenschutz“ erfüllen und - soweit ersichtlich - trotz unterschiedlicher fachärztlicher Ausbildung wohl auch in der Lage sind, die zur Feststellung der rechtfertigenden radiologischen Indikation erforderlichen Angaben zu ermitteln und an den Teleradiologen der Radiologischen Gemeinschaftspraxis weiterzuleiten so- wie die Patienten aufzuklären." (Bayer. VGH, Urt. v. 14.04.2008, Az.: 9 B 08.81)

Seitens der zuständigen Genehmigungsbehörden für die Teleradiologie nach der RöV werden hinsichtlich der Ärzte nach $\S 3$ Abs. 4 S. 1 Nr.3 RöV gelegentlich konkrete Zahlenvorgaben gemacht, die z.B. von der entsprechenden Einrichtung zu erfüllen sind. Ebenso wird teilweise von den Behörden gefordert, dass zur Erfüllung dieser Anforderungen in Krankenhäusern grundsätzlich nur die Ärzte im Vordergrunddienst mitgezählt werden dürfen, nicht jedoch z.B. Oberärzte im Hintergrunddienst. Für derartige Vorgaben der Genehmigungsbehörden existieren jedoch weder in der RöV, noch in anderen Vorschriften (z.B. den Weiterbildungsordnungen der Landesärztekammern) entsprechende Regelungen. Gefordert wird nach der RöV nur, dass im Verhältnis zur Größe der Einrichtung bzw. des Krankenhauses genügend Ärzte nach $\S 3$ Abs. 4 S. 1 Nr. 3 RöV zur Verfügung stehen.

Im Übrigen ist den Ausführungen des Bayerischen Verwaltungsgerichtshofs in dem oben zitierten Urteil zu entnehmen, dass Teilgebietsradiologen, die in ihrem jeweiligen Teilgebiet die Fachkunde im Strahlenschutz besitzen, nicht zusätzlich die erforderlichen Kenntnisse im Strahlenschutz nach §3 Abs. 4 S. 1 Nr. 3 RöV gesondert nachweisen müssen.

Verfassungswidrigkeit des Regionalprinzips nach $\S 3$ Abs. 4 S. 1 Nr. 6 RöV?

Fraglich ist, inwieweit die Anforderung des §3 Abs.4 Nr.6 RöV erfüllt sein musS.Danach muss der Teleradiologe innerhalb eines für die Notfallversorgung erforderlichen Zeitraums am Ort der technischen Durchführung eintreffen. Darunter wird ein Zeitraum von 45 Minuten verstanden. Dieser räumliche Zusammenhang zwischen dem Teleradiologen und dem Ort der Untersuchung wird auch als „Regionalprinzip“ bezeichnet. Diese Regelung ist vom Verwaltungsgericht Köln in einem Urteil vom 28.01.2010 (Az.: $13 \mathrm{~K}$ 1158/06) für unverhältnismäßig und damit im Ergebnis für verfassungswidrig erachtet worden.

Das Verwaltungsgericht führt hierzu aus, dass sich herausgestellt hätte, dass es im Rahmen der Teleradiologie keinerlei Szenarien gebe, in denen dieses zeitliche Er- 
fordernis zwingend eingehalten werden müsse. Bei Schwangeren und Kindern würden keine teleradiologischen Maßnahmen durchgeführt. Kontrastmittelzwischenfälle werden vom Verwaltungsgericht nicht als radiologische, sondern als internistische Notfälle angesehen, da sich der Internist um Kontrastmittelunverträglichkeiten kümmern müsse. Die Gefahr, dass der Teleradiologe durch Bewusstlosigkeit oder Tod ausfalle, sei mehr als unwahrscheinlich. Zudem könne dieser Gefahr durch das Vorhandensein eines weiteren Teleradiologen begegnet werden. Die Bilder selber könnten hingegen nicht verloren gehen, da sie vor Ort abgespeichert werden, jederzeit vorhanden seien und im äußersten Notfall ausgedruckt und zu einem im Umkreis ansässigen Radiologen gebracht werden könnten. Ein Ausfall aller Kommunikationssysteme sei zudem kaum denkbar. In jedem Fall müsse der Patient keiner erneuten Strahlenbelastung ausgesetzt werden.

Bereits die Prämisse des VG Köln, wonach die Regelung in §3 Abs. 4 Nr. 6 RöV unverhältnismäßig sei, da Notfallsituationen im Rahmen der teleradiologischen Leistungserbringung nicht bekannt seien, die das Erfordernis der Genehmigungsvoraussetzung rechtfertigen würden, erscheint zweifelhaft und auch im Detail nicht zutreffend. Darüber hinaus hat sich das VG Köln nicht mit dem weiteren gesetzgeberischen Ziel dieser Regelung, dem Entgegenwirken einer überregionalen Ausweitung teleradiologischer Kommunikationssysteme auseinandergesetzt. Nach Ansicht des Verordnungsgebers sind auch bei einem teleradiologischen Betrieb „persönliche Kontakte zwischen dem Arzt mit der erforderlichen Fachkunde im Strahlenschutz und dem Arzt am Ort der Untersuchung zum Nachteil des Patienten“ erforderlich (Wigge P, Kaiser R, Fischer J, Loose R. MedR 2010; 700, 706). Das Regionalprinzip ist im Ergebnis daher nicht unverhältnismäßig, da es dem Umstand Rechnung trägt, dass unter der Prämisse eines bestimmten typischen - ausgedünnten - Versorgungsbilds, nämlich der Gegebenheiten während der Nacht sowie an Wochenend- und Feiertagen, „die Anwesenheit des indizierenden Arztes beim Patienten zumindest in personeller Hinsicht gemindert“, jedoch nicht vollständig aufgehoben werden soll (vgl. OVG NRW Beschluss vom 18.12.2007, Az.: 20 A 943/07). Das Oberverwaltungsgericht des Landes Nordrhein-Westfalen (OVG NRW) hat in dem Berufungsverfahren (Az.: $20 \mathrm{~A}$
497/10) gegen das Urteil des VG Köln daher ebenfalls die Auffassung vertreten, dass das Regionalprinzip rechtmäßig und verfassungskonform ist, auch wenn das Verfahren nicht mit einem Urteil, sondern mit einem Vergleich beendet worden ist (vgl. Wigge P, Tonner U. Fortschr Röntgenstr 2012; 184: 176-180).

Auch das Verwaltungsgericht Aachen hat beispielsweise in dem oben genannten Urteil vom 08.02.2007 (Az.: 6 K 276/06) die Notwendigkeit der örtlichen Nähe des Teleradiologen betont, um einer Ausweitung der Teleradiologie eine Absage zu erteilen. Auch in der Literatur wird das Regionalprinzip im Sinne einer optimalen Patientenversorgung für verfassungsmäßig erachtet, weil darauf abgestellt wird, dass in Notfallsituationen die Spezialkenntnisse des Radiologen ggf. erforderlich seien (Rosenberg C, Langner S, Rosenberg B, Hosten N. Fortschr Röntgenstr 2011; 183: 804, 807).

\section{Patientenrechtegesetz und Telera- diologie \\ $\nabla$}

Ein Wertungswiderspruch zu den Vorschriften für die Teleradiologie in der RöV ergibt sich scheinbar aus den Regelungen des Patientenrechtegesetzes vom 20.03.2013. Die durch das Patientenrechtegesetz eingeführte Bestimmung über den sog. Behandlungsvertrag nach $\S 630 \mathrm{e}$ des Bürgerlichen Gesetzbuches (BGB) hat folgenden Inhalt:

„(1) Der Behandelnde ist verpflichtet, den Patienten über sämtliche für die Einwilligung wesentlichen Umstände aufzuklären. Dazu gehören insbesondere Art, Umfang, Durchführung, zu erwartende Folgen und Risiken der Maßnahme sowie ihre Notwendigkeit, Dringlichkeit, Eignung und Erfolgsaussichten im Hinblick auf die Diagnose oder die Therapie. Bei der Aufklärung ist auch auf Alternativen zur Maßnahme hinzuweisen, wenn mehrere medizinisch gleichermaßen indizierte und übliche Methoden zu wesentlich unterschiedlichen Belastungen, Risiken oder Heilungschancen führen können.

\section{(2) Die Aufklärung muss}

1. mündlich durch den Behandelnden oder durch eine Person erfolgen, die über die zur Durchführung der Maßnahme notwendige Ausbildung verfügt; ergänzend kann auch auf Unterlagen Bezug ge- nommen werden, die der Patient in Textform erhält, (...)“.

Hier liegt scheinbar ein Widerspruch zu der Rolle der beiden beteiligten Ärzte im Rahmen der Teleradiologie vor, wonach der Teleradiologe, der die rechtfertigende Indikation stellt und die Befundung vornimmt, nicht die Aufklärung des Patienten vornimmt. Zu dem Verhältnis dieser Normen hat sich die Rechtsprechung bisher nicht geäußert.

Unterschiedliche Zielrichtungen der Röntgenverordnung und des Patientenrechtegesetzes

Zunächst ist anzumerken, dass die Vorschriften in der RöV und dem BGB unterschiedliche Zielrichtungen haben:

Die Röntgenverordnung regelt öffentlichrechtlich im Sinne der Gefahrenabwehr die Grundsätze der Anwendung von Röntgenstrahlung auf den Menschen und beschreibt, wann und in welcher Form die Anwendung von Röntgenstrahlen erlaubt und ggf. genehmigungsfähig ist.

In den Neuregelungen zum Behandlungsvertrag nach den $\S \S 630$ a ff. BGB aufgrund des Patientenrechtegesetzes ist dagegen zivilrechtlich festgelegt worden, welche Pflichten der Behandelnde (Arzt, Heilberufe) gegenüber dem Patienten hat und wann es privatrechtlich zu einem Haftungsanspruch des Patienten gegen den Behandelnden kommen kann. Die Regelungen wirken daher nur im Verhältnis zwischen dem Behandelnden und dem Patienten aufgrund des Behandlungsvertrags.

Auslegung des $\$ 630$ e BGB und Verhältnis zu §3 Abs. 4 RöV

$\S 630$ e Abs.2 S. 1 Nr.2 BGB verlangt die Aufklärung durch den Behandelnden oder eine Person, die über die zur Durchführung der Maßnahme notwendige Ausbildung verfügt. Daher besteht die Frage, wie die Formulierung „zur Durchführung der Maßnahme notwendige Ausbildung“ auszulegen ist.

Nach ständiger Rechtsprechung kann die Aufklärung auf einen anderen Arzt delegiert werden (BGH, Urt. v. 27.11.1973; Az.: IV ZR 167/72). Behandelnder und aufklärender Arzt können daher auseinanderfallen (Schreiber, in: Hk-BGB, §630e BGB, Rn. 3). Nach der Gesetzesbegründung zum 
Patientenrechtegesetz muss dieser aufklärende Arzt die notwendige theoretische Befähigung für die Behandlung haben, nicht jedoch die erforderliche praktische Erfahrung für die selbstständige Durchführung der Maßnahme selbst (BTDrS. 17/11710, S. 38 f.). Der Arzt, auf den die Aufklärung übertragen wurde, muss nicht die Kenntnisse eines Facharztes haben; er muss jedoch die für eine ordnungsgemäße Aufklärung notwendigen Kenntnisse aufweisen, was auch Kenntnisse erfordern kann, die über den Facharztstandard hinausgehen (Bender, VersR 2013, S. 962, Weidenkaff, in: Palandt, BGB, $\S 630$ e BGB, Rn. 8). Maßgeblich wird in der Literatur hinsichtlich der „Ausbildung“ auf die ärztliche Approbation abgestellt (Rehborn, GesR 2013, S.257, 264 mit Bezugnahme auf BVerfG, Beschl. V. 01.02.2011; Az.: 1 BvR 2383/10). Entscheidend ist somit nicht die formale Qualifikation als Facharzt, sondern die Fähigkeit, den Patienten zu informieren und dessen Fragen sachgerecht zu beantworten (vgl. Rehborn, a.a.O.).

Dies widerspricht jedoch grundsätzlich nicht dem Regelungssystem der RöV hinsichtlich der Teleradiologie. Im Rahmen der teleradiologischen Untersuchung sind die Leistungsanteile räumlich auf verschiedene Ärzte aufgeteilt, wenngleich der Hauptanteil beim Teleradiologen gemäß §3 Abs. 4 Nr. 1 RöV liegt, da dieser die rechtfertigende Indikation zu stellen hat und die Befundung durchführt.

Nur der Teleradiologe hat nach §24 Abs. 1 Nr. 1 RöV „für das Gesamtgebiet der Röntgenuntersuchung oder Röntgenbehandlung die erforderliche Fachkunde im Strahlenschutz", die notwendig ist, um nach §23 RöV die rechtfertigende Indikation zu stellen; allerdings muss auch der Arzt am Ort der technischen Durchführung die gemäß §3 Abs. 4 S. 1 Nr. 3 RöV erforderlichen Kenntnisse im Strahlenschutz aufweisen, um die notwendigen Informationen zur rechtfertigenden Indikation zu ermitteln und den Patienten wirksam aufzuklären. Mithin weist er, wie von der Rechtsprechung im Rahmen der Aufklärung gefordert, Kenntnisse auf, um den Patienten in sachgerechter Form aufzuklären.

Zudem ist zu bemerken, dass $§ 630$ e BGB zwar die neuere Vorschrift ist, §3 Abs. 4 RöV jedoch eine sogenannte lex specialis hinsichtlich der Durchführung der Teleradiologie sein dürfte. Allerdings ist der Teleradiologe, wie jeder Behandelnde, verpflichtet, sicherzustellen, dass der aufklärende Kollege die entsprechenden Kenntnisse für eine ordnungsgemäße Aufklärung besitzt (BGH, Urt. v. 07.11.2006; Az.: VI ZR 206/05; Weidenkaff, in: Palandt, BGB, §630 e BGB, Rn. 8).
Fazit $\nabla$

Im Rahmen der mit den Genehmigungsbehörden strittigen Fragen zur Teleradiologie gibt es bisher kaum Äußerungen der Obergerichte, sondern nur einzelne verwaltungsgerichtliche Urteile. Gleichwohl können diese hinsichtlich der Genehmigungsvoraussetzungen und des Arzteinsatzes hilfreiche Argumentationsansätze für Ärzte, Krankenhausträgern und Betreiber gegenüber den Behörden liefern.

In der Rechtsprechung noch ungeklärt ist das Verhältnis der RöV zu den Vorschriften des PatientenrechtegesetzeS.Es spricht jedoch einiges dafür, dass die bisherigen Verfahrensweisen in der Teleradiologie dem Patientenrechtegesetz nicht zuwider laufen.

Prof. Dr. Peter Wigge

Rechtsanwalt, Fachanwalt für Medizinrecht

Lic. iur. can. Urs Fabian Frigger Rechtsanwalt

Rechtsanwälte Wigge

Scharnhorststr. 40

48151 Münster

Telefon: (0251) 53595-0

Telefax: (0251) 53595-99

E-Mail: kanzlei@ra-wigge.de

www.ra-wigge.de 